\title{
Mitochondrial DNA variation and phylogeography of native Mongolian goats
}

\author{
Onolragchaa Ganbold ${ }^{1,2}$, Seung-Hwan Lee ${ }^{1}$, Woon Kee Paek³, Munkhbaatar Munkhbayar², \\ Dongwon Seo ${ }^{1}$, Prabuddha Manjula', Tamir Khujuu ${ }^{2}$, Erdenetushig Purevee ${ }^{2}$, and Jun Heon Lee ${ }^{1, *}$
}

\author{
* Corresponding Author: Jun Heon Lee \\ Tel: +82-42-8215779, Fax: +82-42-8215779, \\ E-mail: junheon@cnu.ac.kr \\ ${ }^{1}$ Laboratory of Animal Molecular Genetics, Division \\ of Animal \& Dairy Science, Chungnam National \\ University, Daejeon 34134, Korea \\ 2 Department of Biology, Mongolian National \\ University of Education, Ulaanbaatar 210685, \\ Mongolia \\ ${ }^{3}$ Daegu National Science Museum of Korea, Daegu \\ 43014, Korea

\section{ORCID} \\ Onolragchaa Ganbold \\ https://orcid.org/0000-0002-8480-3630 \\ Seung-Hwan Lee \\ https://orcid.org/0000-0003-1508-4887 \\ Woon Kee Paek \\ https://orcid.org/0000-0002-4587-1024 \\ Munkhbaatar Munkhbayar \\ https://orcid.org/0000-0002-7977-5110 \\ Dongwon Seo \\ https://orcid.org/0000-0003-0548-7068 \\ Prabuddha Manjula \\ https://orcid.org/0000-0001-8074-8323 \\ Tamir Khujuu \\ https://orcid.org/0000-0002-9178-2583 \\ Erdenetushig Purevee \\ https://orcid.org/0000-0002-9691-8670 \\ Jun Heon Lee \\ https://orcid.org/0000-0003-3996-9209
}

Submitted May 13, 2019; Revised Jul 5, 2019; Accepted Sept 15, 2019
Objective: Mongolia is one of a few countries that supports over 25 million goats, but genetic diversity, demographic history, and the origin of goat populations in Mongolia have not been well studied. This study was conducted to assess the genetic diversity, phylogenetic status and population structure of Mongolian native goats, as well as to discuss their origin together with other foreign breeds from different countries using hypervariable region 1 (HV1) in mtDNA.

Methods: In this study, we examined the genetic diversity and phylogenetic status of Mongolian native goat populations using a 452 base-pair long fragment of HVI of mitochondrial DNA from 174 individuals representing 12 populations. In addition, 329 previously published reference sequences from different regions were included in our phylogenetic analyses.

Results: Investigated native Mongolian goats displayed relatively high genetic diversities. After sequencing, we found a total of 109 polymorphic sites that defined 137 haplotypes among investigated populations. Of these, haplotype and nucleotide diversities of Mongolian goats were calculated as $0.997 \pm 0.001$ and $0.0283 \pm 0.002$, respectively. These haplotypes clearly clustered into four haplogroups (A, B, C, and D), with the predominance of haplogroup A (90.8\%). Estimates of pairwise differences (Fst) and the analysis of molecular variance values among goat populations in Mongolia showed low genetic differentiation and weak geographical structure. In addition, Kazakh, Chinese (from Huanghuai and Leizhou), and Arabian (Turkish and Baladi breeds) goats had smaller genetic differentiation compared to Mongolian goats. Conclusion: In summary, we report novel information regarding genetic diversity, population structure, and origin of Mongolian goats. The findings obtained from this study reveal that abundant haplogroups (A to D) occur in goat populations in Mongolia, with high levels of haplotype and nucleotide diversity.

Keywords: Genetic Diversity; Mitochondrial DNA; Native Goats; Phylogenetic Relationships; The Origin

\section{INTRODUCTION}

The domestic goat (Capra hircus) is one of the earliest domesticated animals. Archaeological evidence suggests that the Fertile Crescent region in the Near East (NE) was the domestication center of domestic goats, and that this event occurred more than 10,000 years ago $[1,2]$. Meanwhile, some studies suggest a second, independent domestication event for Cashmere-like goat breeds in Pakistan [3]. Recently, goat breeds are recognized as one of the most important (source of meat, milk, and fiber) and adaptable (adapted to savannas, desert, high mountain, and cold winters) domestic animals in the world, but particularly in countries of Africa and Asia. Three wild species of Capra, bezoar (C. aegagrus), ibex (C. ibex) and markhor (C. falconeri) are phylogenetically close to the domestic type (C. hircus), 
and it has been suggested that all these wild species contributed to the genetic composition of domestic goats [4-6]. Among these candidates, C. aegagrus is widely agreed to be the wild ancestor of domestic goats, as all haplogroups of domestic goat occur within current C. aegagrus populations $[6,7]$.

Mitochondrial DNA (mtDNA) is a critical tool for tracing evolutionary and population genetics of most domesticated animals. Also, mtDNA diversity is vital in assessing the maternal origin, phylogeny, and population structure of all domestic animals [8-13]. Previous studies of goat mtDNA (control region) revealed a total of six haplogroups (also known as lineages; A, B, and C by Luikart et al [14]; D by Sultana et al [15]; F by Sardina et al [16]; and G by Naderi et al [6]). Of these, haplogroup $\mathrm{A}$ is the most widely distributed through the world (approximately $90 \%$ of total goats belong to A haplogroup), while rest of haplogroups (approximately $10 \%$ of total goats) have limited distribution areas with $B$ haplogroups occurs only in southern and eastern Asia [17]. These previous studies [6,14-16] also revealed their relatively weak phylogeographic structure across the world.

Due to their high economic importance, goats are considered as an important livestock species in Mongolia. In 2017, the estimated population of goats in Mongolia was 27.3 million, $41.3 \%$ of the total of 66.7 million heads of livestock [18]. All goats in Mongolia are reared as a source of cashmere in the country, while they are used relatively less as a source of milk and meat. For this reason, Mongolia is a major cashmere producer (Mongolia is the world's second largest cashmere producer behind China), it produces about $30 \%$ of the world's cashmere [19]. There are eight goat strains/breeds recognized in Mongolia [20,21], and they are adapted well to various natural zones including forest, steppe, desert, and high-mountain. Unfortunately, little is known about the genetic diversity, phylogenetic relationships, and origins of goat populations in Mongolia. Only a few samples were included in merged datasets of previous studies that explored the population structure of world goat populations $[6,14,22]$. We therefore initiated a study of partial mtDNA sequences to evaluate the genetic diversity and origin of Mongolian native goat (MG). Then, the main objective of this study was to assess the genetic diversity, phylogenetic status and population structure of MG, as well as to discuss their origin together with other foreign breeds from different countries using hypervariable region 1 (HV1) in mtDNA.

\section{MATERIALS AND METHODS}

\section{Animal care}

All sampling procedures in this study were carried out in accordance with the recommendations of "Guide for Care and Use of Agricultural Animals in Research and Teaching, 3rd edition" published by the Federation of Animal Science Soci- eties (ISBN: 978-1-884706-11-0) and also "Guidelines for sampling and handling animals for purposes of Veterinary and Other researches" of Livestock sector of Mongolia, with full approval from Committee Ministry of Agriculture and Food of Mongolia (ref. A67/2010, in Mongolian). The protocol approved by Committee of Ministry of Agriculture and Food of Mongolia was used in this study. Animal owners had informed consent for inclusion of their animals in the study, and they involved sampling procedures.

\section{Animals and DNA extraction}

Sampling was conducted across Mongolian territory in 2017 and 2018. In this study, a total of 158 unrelated genetic samples that consisted of 97 blood (2017), 10 ear skin, and 51 tail hair (2018) were gathered from 11 different populations (Figure 1A). We collected different samples during sampling years due to availability of field equipment. Of 11 populations, two populations were recognized as separate native strains (Zalaa Jinstiin White; MZG and Gobi-Gurwan Saikhan Black; MGG), while the other nine populations were believed to be Mongolian indigenous native goats (MNG) (Table 1, Figure 1A). Geographical distances between sampling sites ranged from $\sim 65 \mathrm{~km}$ to $\sim 1,450 \mathrm{~km}$. When selecting animals from each investigated population, we involved owners to avoid sampling from related animals. Genomic DNA was extracted from punches of filter paper (FTA classic cards Whatman: Lot No.9767339) using a MagMax DNA Multi-Sample Ultra Kit, while genomic DNA was extracted from ear tissue and hair samples using a DNeasy Blood and Tissue Kit (QIAGEN, Hidden, Germany) following the recommended manufacturer's instructions.

\section{Polymerase chain reaction amplification and sequencing}

To study genetic diversity and phylogenetic analyses of MG, we amplified a $452 \mathrm{bp}$ mtDNA fragment of the caprine partial HV1 region in D-loop (positions 15,678 to 16,130 on the complete goat mitochondrial sequence of reference $\mathrm{NC}_{-}$ 005044) from 158 animals using a set of primers: gd-loop $F$ (5'-CAC AAA CTT CCC ACT CCA CA -3') and gd-loop R (5'-AGC GTG TTT AAA ACG GTG GT -3'). We designed these two internal primers using the online tool Primer3Plus (online available at: http://www.bioinformatics.nl/cgi-bin/ primer3plus/primer3plus.cgi) based on a reference sequence (accession number: NC_005044). Polymerase chain reaction (PCR) amplifications were performed on a total of 20 $\mu \mathrm{L}$ final volume that included $2.0 \mu \mathrm{L}$ genomic DNA (20 $\mathrm{ng} / \mu \mathrm{L}), 0.8 \mu \mathrm{L}$ of each primer (10 pmoles $/ \mu \mathrm{L}), 2.0 \mu \mathrm{L} 10 \times$ reaction buffer, $1.6 \mu \mathrm{L}$ dNTP, $0.2 \mu \mathrm{L}$ prime Taq DNA polymerase, and $12.6 \mu \mathrm{L}$ distilled water to adjust the final PCR product volume. The reaction conditions for amplification consisted of initial denaturation at $94^{\circ} \mathrm{C}$ for $3 \mathrm{~min}$, followed 
Table 1. Estimated genetic diversities in Mongolian native goat (Capra hircus) populations and their haplogroup distributions

\begin{tabular}{|c|c|c|c|c|c|c|c|c|}
\hline Populations & ID & $n^{1)}$ & $h^{2)}$ & $H \mathrm{~d}^{3)}$ & $S^{4)}$ & $\mathrm{Pi}^{5)}$ & $\mathrm{K}^{6)}$ & $H^{7)}(n)$ \\
\hline Altai & ALT & 11 & 10 & $0.982 \pm 0.046$ & 65 & $0.0384 \pm 0.009$ & 17.34 & $A(9), C(1), D(1)$ \\
\hline Baganur & BGN & 20 & 19 & $0.995 \pm 0.018$ & 41 & $0.0201 \pm 0.001$ & 9.068 & $A(20)$ \\
\hline Bogd & $\mathrm{BOG}$ & 16 & 14 & $0.983 \pm 0.028$ & 39 & $0.0213 \pm 0.004$ & 9.608 & $A(15), D(1)$ \\
\hline Erdenet & ERD & 10 & 7 & $0.933 \pm 0.062$ & 31 & $0.0217 \pm 0.003$ & 9.800 & $A(10)$ \\
\hline Ikh Nart & INT & 23 & 21 & $0.992 \pm 0.015$ & 69 & $0.0319 \pm 0.005$ & 14.371 & $A(19), C(1), D(3)$ \\
\hline Mankhan & MAN & 20 & 18 & $0.989 \pm 0.019$ & 69 & $0.0329 \pm 0.006$ & 14.563 & $A(17), C(1), D(2)$ \\
\hline Lun Og & LOG & 10 & 9 & $0.978 \pm 0.054$ & 42 & $0.0355 \pm 0.004$ & 16.067 & $A(8), D(2)$ \\
\hline Lun Tsag & LTS & 18 & 13 & $0.961 \pm 0.030$ & 36 & $0.0199 \pm 0.001$ & 8.993 & $A(18)$ \\
\hline Nomgon & NOM & 10 & 8 & $0.956 \pm 0.059$ & 53 & $0.0334 \pm 0.009$ & 15.089 & $A(9), C(1)$ \\
\hline Bulgan & MGG & 10 & 10 & $1.0 \pm 0.045$ & 53 & $0.0321 \pm 0.011$ & 14.533 & $A(9), C(1)$ \\
\hline Jinst & MZG & 10 & 10 & $1.0 \pm 0.045$ & 31 & $0.0211 \pm 0.001$ & 9.533 & $A(10)$ \\
\hline Ref. & Ref. $^{8)}$ & 16 & 16 & $1.0 \pm 0.022$ & 72 & $0.0368 \pm 0.007$ & 16.63 & $A(14), B(1), C(1)$ \\
\hline Overall & 12 & 174 & 137 & $0.997 \pm 0.001$ & 109 & $0.0283 \pm 0.002$ & 12.75 & $A(158), B(1), C(6), D(9)$ \\
\hline
\end{tabular}

1) Number of animals. ${ }^{2)}$ Number of haplotypes. ${ }^{3)}$ Haplotype diversity. ${ }^{4)}$ Number of segregating sites.

${ }^{5)}$ Nucleotide diversity. ${ }^{6)}$ Average number of nucleotide differences.

${ }^{7)}$ Identified haplogroups in each population (number of animals).

${ }^{8)}$ Animals from Luikart et al [14] as a reference population.

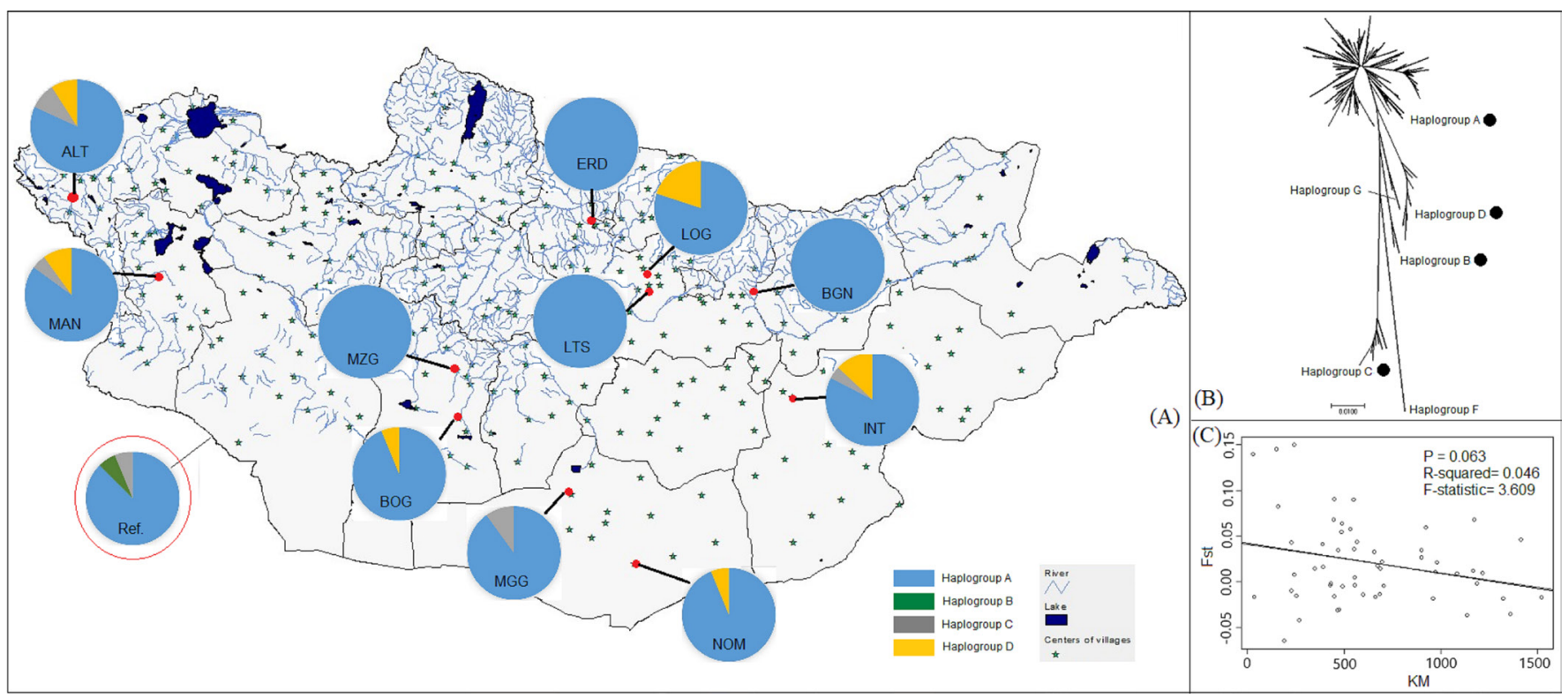

Figure 1. (A) Sampling localities of 12 Mongolian native goat (MG) populations (population ID presented): red spots indicating exact sampling locations, Ref. population located outside without specific location. (B) Un-rooted neighbor-joining tree that illustrating four haploroups of MG (black spots). And (C) Regression plot of relationships of genetic (Fst) and geographic ( $\mathrm{km})$ distances.

by 35 cycles with denaturation for $30 \mathrm{~s}$ at $94^{\circ} \mathrm{C}$, annealing at $55^{\circ} \mathrm{C}$ for $30 \mathrm{~s}$, extension for $1 \mathrm{~min}$ at $72^{\circ} \mathrm{C}$, and final extension at $72^{\circ} \mathrm{C}$ for $5 \mathrm{~min}$. All PCRs ran with negative controls and were performed by a thermal cycler C1000 system (BioRad laboratories Inc., Hercules, CA, USA). After amplification, approximately $5 \mu \mathrm{L}$ of $\mathrm{PCR}$ product was run on $1.5 \%$ agarose gel at a constant voltage of $150 \mathrm{~V}$ for $30 \mathrm{~min}$ in $1 \times \mathrm{TAE}$ buffer prior bands and size checked under UV light. A service provider company (GenoTech Corp. Deajeon, Korea) performed direct sequencing of purified PCR products using a Sanger DNA sequencing approach in an ABI Prime 3730 DNA sequence machine. All 158 novel sequences of Mongolian goats in this study were deposited in the Genbank public database under accession numbers MK165491MK165648.

\section{Data assemble and analysis}

A total of 158 sequences representing 11 MG populations 
from this study were merged with the previously described 16 sequences (considered as separated populations) for total of $12 \mathrm{MG}$ populations. To examine the phylogenetic status and the origin of MG, 174 sequences of Mongolian goats were initially edited with BioEdit v.7.2.5 [23] and partial sequences aligned positions using the ClustalW tool [24] together with an additional 329 reference sequences of 24 foreign breeds from different geographic regions in the Old World and three wild caprine species, as well as representative sequences of the previously defined six caprine haplogroups $(\mathrm{A}, \mathrm{B}, \mathrm{C}, \mathrm{D}$, $\mathrm{F}$, and $\mathrm{G}$ ) (accession numbers of all reference sequences in Supplementary Table S1).

At the individual population level, as well as all MG together, DNA Sequence Polymorphism v5.10.01 (DnaSP [25]) was used to calculate the following diversity parameters of mtDNA: number of haplotypes $(\mathrm{h})$, haplotype diversity $(\mathrm{Hd})$, number of segregating sites (S), nucleotide diversity $(\mathrm{Pi})$, and average number of nucleotide differences $(\mathrm{K})$.

We constructed a neighbor-joining (NJ) tree (using 500 bootstraps to determine evolutionary distances computed based on the Tamura-Nei distance model; [26]) to examine possible haplogroups in MG populations and phylogenetic relationships among MG populations, as well as other foreign breeds using Molecular Evolutionary Genetic Analysis version.7.0.21 (MEGA [27]). The genetic distance (Tamura-Nei) between $12 \mathrm{MG}$ populations were also assessed using MEGA. The Roehl network data ( ${ }^{*}$.rdf) files for network analysis were generated by DnaSP. Then, we drew the Median-Joining (MJ) network using NETWORK v.5.0.0.1 [28] to trace genetic relationships among identified haplotypes within MGs and between other breeds from different regions.

Arlequin v3.5 [29] was used to estimate pairwise genetic distance (significant values were accepted when $\mathrm{p}<0.05$ ), average number of pairwise differences between and within populations, and analysis of molecular variance (AMOVA) to assess the population genetic structure (with 1,000 permutations). We used, and explored in Arlequin v3.5, two main approaches: i) Fu's (1997) neutrality tests of $F s$ and ii) mismatch distribution analysis [30], to assess signs of historical population expansions. Two tests of goodness of fit, sum of squared deviation (SSD) and Harpending's raggedness index (r) [31], were used to evaluate the significance of deviations within observed patterns of mismatch from the simulated model of demographic expansion ( $\mathrm{p}$ value accepted at $<0.05$ ).

\section{RESULTS}

mtDNA variation and genetic diversity

Comparison of 174 sequences of the HVI region of goats from 12 different Mongolian goat populations showed high polymorphism. There were 109 polymorphic sites over $452 \mathrm{bp}$, while only two insertion/deletion (InDel) mutations were detected. Estimated diversity parameters for MG populations are given in Table 1. Among these polymorphic sites, we identified 20 singletons and 89 parsimony informative sites that defined 137 haplotypes (three of these haplotypes defined by two indels) with a relatively high $H d$ (overall; $0.997 \pm 0.001$ ). Estimated values of $H \mathrm{~d}$ ranged from 0.933 (Erdenet; ERD) to the maximum value of 1.000 (MGG, MZG, and Ref), while nucleotide diversity $(\mathrm{Pi})$ varied between 0.0199 (Lun Tsag; LTS) and 0.0384 (Altai; ALT) (overall, Pi $=0.0283$ ) (Table 1). The mean $\mathrm{K}$ was 12.75 , and the highest $\mathrm{K}$-value was observed for the ALT population (17.34). In addition, an average base composition was $\mathrm{A}=31.0 \%, \mathrm{~T}=30.3 \%, \mathrm{G}=16.5 \%$, and $\mathrm{C}=$ $22.2 \%$ in a $452 \mathrm{bp}$ HVI region. Thus, the percentage of $\mathrm{A}+\mathrm{T}$ (61.3\%) nucleotide pairs was higher than the nucleotide pairs of $\mathrm{C}+\mathrm{G}(38.7 \%)$.

\section{Phylogeography of Mongolian goats}

Phylogenetic relationships of goat populations within Mongolian and between 36 breeds (MG plus foreign breeds) were initially investigated in this study based on the high polymorphic HVI region in mtDNA. Because previous studies $[6,11]$ have described two origins for haplogroups of domestic goat (one for B, remaining one same for other five haplogroups), haplogroups in MG were identified through an un-rooted $\mathrm{NJ}$ tree using representative sequences of six haplogroups to discern possible origin for MG in this study. More in detail, the NJ tree clearly divided all MG sequences into four different haplogroups: A, B, C, and D (Figure 1B). The findings suggested two origins for the MG population. Among these, haplogroup A was highly predominant and found in all populations of MG (100\% for ERD, LTS, Baganuur; BAG, and MZG). In contrast, relatively low numbers of animals belonged to haplogroups $C$ (6/12 populations) and $D(5 / 12)$, and only one for haplogroup B (1/12) (Table 1, Supplementary Table S2). Thus, the frequencies of haplogroups A, B, C, and $\mathrm{D}$ in $\mathrm{MG}$ populations were $90.8 \%, 0.57 \%, 3.4 \%$, and $5.2 \%$, respectively. Furthermore, ALT, Ikh Nart (INT), and Mankhan (MAN) populations were found to contain three haplogroups (A, C, and D), while there was no population with all four haplogroups (Table 1). To obtain further insight into the phylogenetic status of $\mathrm{MG}$, a NJ tree was constructed once again for $12 \mathrm{MG}$ populations and a total of 503 sequences that consisted of 36 populations ( 24 foreign and $12 \mathrm{MG}$ ), as well as three species of wild caprine using Tamura-Nei distance model (Figure 2, Supplementary Figure S1). Supplementary Table S3 presents Tamura-Nei genetic distance matrix (within/between) of MG. The results of NJ tree and genetic distance matrix showed relatively close relationships of MG populations, and indicated an absence of population-specific clusters in the NJ tree (Supplementary Figure S1). In addition, goats from China, Korea, and Central Asian (Kazakh and Kyrgyz), and some from Arabia had relatively close relationships com- 




Figure 2. Constructed neighbor-joining tree of domestic (Capra hircus) and wild (genus of Capra) goats based on 452 bp hypervariable region 1 region from 503 sequences (174 MG and 329 reference sequences from different regions).

pared to MG, while goats from Europe, Africa, and South Asia (except some Indian goats) clustered separately (Figure 2). Among the MG populations, Tamura-Nei distance within population reached a maximum estimated value in ALT (0.0402), while LTS accounted for the lowest value (0.0204). The genetic distance values between populations varied between 0.0389 (LOG versus Ref.) and 0.0207 (LTS vs BGN) (mean value was 0.0302) (further information see Supplementary Table S3).
To further discern relationships among identified haplotypes in MG populations and in the total dataset (503 sequences), a MJ network analysis was constructed separately. Results obtained from this analysis also supported four well-resolved haplogroups of MG (Figure 3). The topology of the MJ network illustrated relationships of 355 haplotypes of 503 sequences. As mentioned above, 137 of 355 haplotypes belonged to MG, and the majority of these 137 haplotypes clustered into haplogroup A (160 total haplotypes), with only one haplotype 


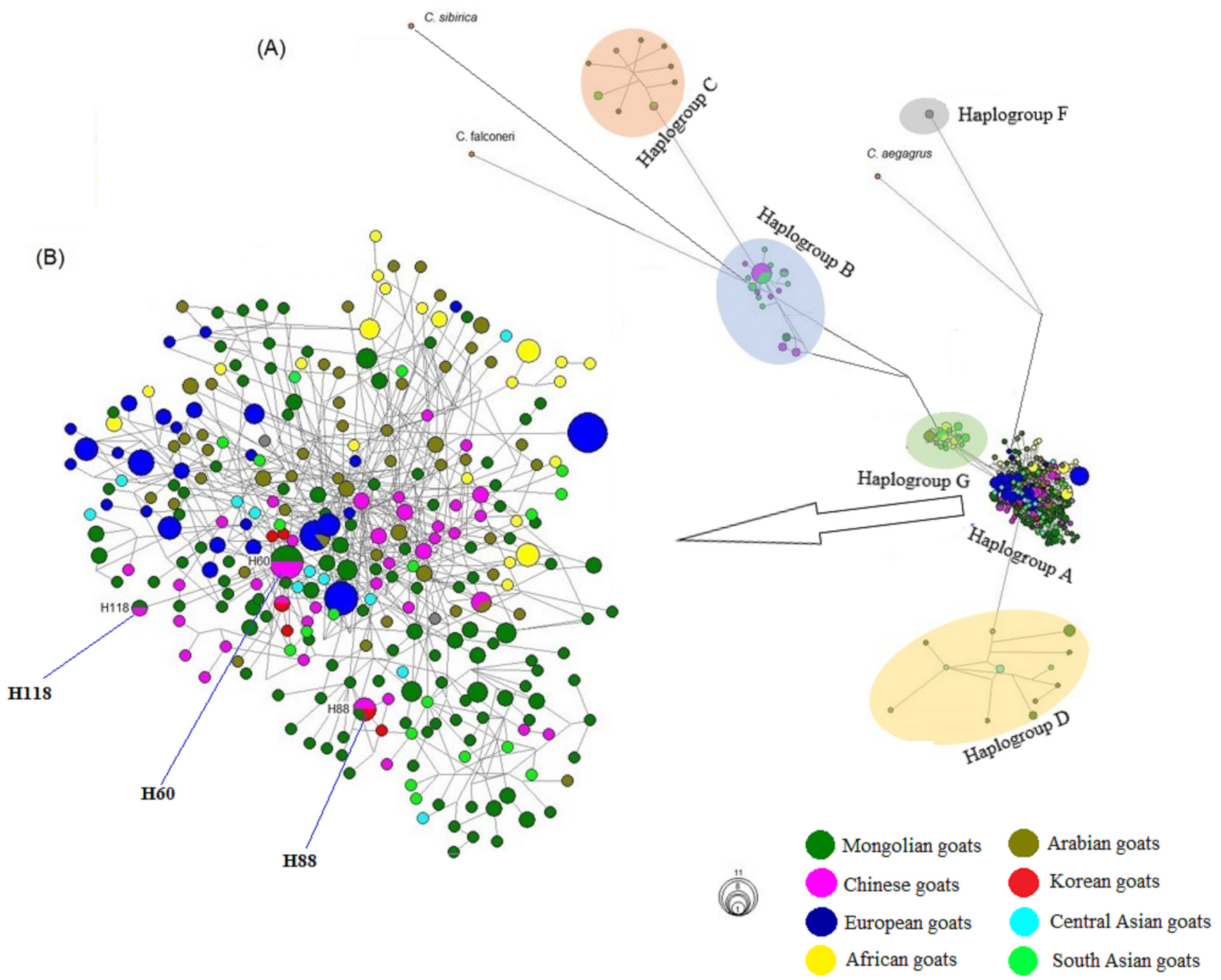

Figure 3. Median-joining network analysis of hypervariable region 1 region from native goats (Capra hircus) from Mongolia (populations, $n=12$ ), and foreign countries (populations, $n=24$ ). (A) network topology of six caprine haplogroups, and (B) predominant haplogroup A with highlighted three haplotypes that shared by Mongolian native goat and other foreign breeds.

for haplogroup B, six haplotypes in haplogroup C, and seven haplotypes in haplogroup D (Figures 1B, 3). Among MG, 121 of 137 haplotypes were unique for a single population, whereas only $16(11.6 \%)$ haplotypes were shared between two or three MG populations, and H19 and H60 were observed as the most frequent haplotypes with four animals (Figure 3, Supplementary Tables S2, S4). In addition, among 355 haplotypes, the three haplotypes that clustered into haplogroup A (H60, H88, and H188) were shared by Mongolian goats and goats from China (seven goats) and Korea (one goat) (Figure 3). Interestingly, Figure 3 also revealed lack of haplotypes that shared between multiple populations or breeds from different regions.

Genetic differentiation and population structure

Additionally, we calculated Wright's F-statistic (pairwise Fst) and average number of pairwise differences between (PiXY) and within $(\mathrm{PiX})$ population values among $12 \mathrm{MG}$ populations, while we also calculated Fst values for comparison among all 36 populations. Estimates of values of genetic differences are indicated in Tables 2 and Supplementary Table S5, respectively. The pairwise Fst values between $12 \mathrm{MG}$ populations revealed relatively low genetic differentiation and ranged between -0.065 and 0.150 (mean Fst $=0.0168$ ). The MGG population was closely related genetically to Nomgon (NOM; $-0.065)$, followed by the distance between NOM and Ref $(-0.0543)$, while comparison of LOG and BGN populations had a greater distance value $(0.150)$ (Table 2$)$. There were no significant relationships between genetic $(F s t)$ and geographical $(\mathrm{km})$ distances among MG populations $\left(\mathrm{R}^{2}=0.04\right.$, d.f $=53, p>0.05$ ) (Figure $1 C$ ). Table 2 also presents the average number of pairwise differences between and within MG 
Table 2. Estimates of pairwise Fst (below) and average number of pairwise differences between (PiXY, above) and within population (PiX, diagonal) among 12 native Mongolian goat (Capra hircus) populations

\begin{tabular}{lcccccrrrrrrr}
\hline Items & INT & LTS & LOG & ERD & MGG & MZG & NOM & BOG & MAN & BGN & ALT & Ref. \\
\hline INT & 15.429 & 12.908 & 16.369 & 13.753 & 15.415 & 12.971 & 15.237 & 12.957 & 15.108 & 12.948 & 16.587 & 16.283 \\
LTS & $0.041^{*}$ & $\mathbf{9 . 2 1 2}$ & 14.796 & 10.480 & 12.222 & 9.330 & 12.505 & 9.897 & 12.564 & 9.327 & 14.586 & 13.769 \\
LOG & 0.016 & $0.140^{*}$ & 16.872 & 15.766 & 17.409 & 14.648 & 17.188 & 14.109 & 16.241 & 14.955 & 17.886 & 18.088 \\
ERD & $0.058^{*}$ & $0.083^{*}$ & $0.146^{*}$ & 10.067 & 13.290 & 10.492 & 13.329 & 10.971 & 13.334 & 9.800 & 14.434 & 14.373 \\
MGG & -0.005 & -0.002 & $0.068^{*}$ & 0.036 & 15.566 & 12.157 & 14.825 & 12.446 & 15.172 & 12.222 & 16.984 & 16.064 \\
MZG & 0.015 & -0.016 & $0.091^{*}$ & 0.055 & -0.042 & 9.766 & 12.495 & 9.682 & 12.519 & 9.364 & 14.539 & 13.773 \\
NOM & -0.031 & 0.005 & 0.044 & 0.022 & -0.065 & -0.031 & 16.006 & 12.794 & 15.111 & 12.312 & 16.644 & 16.004 \\
BOG & 0.017 & 0.034 & 0.064 & $0.090^{*}$ & -0.015 & -0.016 & -0.004 & 9.907 & 12.606 & 9.920 & 14.895 & 13.944 \\
MAN & -0.018 & 0.021 & 0.011 & 0.035 & -0.018 & -0.014 & -0.036 & -0.004 & 15.344 & 12.440 & 16.672 & 16.199 \\
BGN & $0.043^{*}$ & 0.008 & $0.150^{*}$ & 0.015 & -0.004 & -0.016 & -0.014 & 0.033 & 0.010 & 9.289 & 14.280 & 13.566 \\
ALT & -0.017 & $0.068^{*}$ & 0.012 & 0.009 & -0.002 & 0.027 & -0.036 & $0.060 *$ & -0.010 & $0.046^{*}$ & 18.447 & 17.706 \\
Ref. ${ }^{1)}$ & -0.016 & 0.026 & 0.043 & 0.023 & -0.037 & -0.008 & -0.054 & 0.010 & -0.019 & 0.010 & -0.020 & 17.694 \\
\hline
\end{tabular}

${ }^{11}$ Animals from Luikart et al [14] and considered Ref. population in this study.

* Indicates significant Fst $(p<0.05)$.

populations, and mean values were relatively similar, 13.8 and 13.6, respectively. The maximum PiXY value was obtained from a comparison between LOG versus Ref (18.08), and the minimum value was observed between LTS and BGN (9.326). Meanwhile, PiX values ranged from 18.44 (ALT) to 9.21 (LTS). The low genetic differentiation of MG populations was also evidenced by AMOVA analysis in this study. AMOVA revealed poor geographical structure among populations, $98 \%$ of total variation was within populations and only $<2 \%$ was due to differences among populations (Table 3).

In addition, pairwise Fst values from comparisons of 36 breeds from MG and foreign countries and the majority of all possible pairwise Fst comparisons (234 of 288) indicated that MG populations were significantly $(\mathrm{p}<0.05)$ different from foreign populations, in particularly those from Africa, Europe, and some of southern Asia (Iran and Loas) (Supplementary Table S5). However, some populations were closely related to MG populations, in particularly a negative Fst value was observed between Kazakh and Mongolian goats $(-0.190)$, followed by low positive values 0.026 for Indian and MGs, and goats from Arabian countries (including the domestication center).

\section{Population expansion}

We initially studied past demographic dynamics of MG based on two standard approaches of mismatch distribution (number of pairwise differences) and the neutrality test of Fu's [32]
Fs statistic. Because of the small sample size at the individual breed level $(<25)$, these statistics were performed for all datasets of MG and at the haplogroup level (but, haplogroups $\mathrm{B}, \mathrm{C}$, and D were precluded due to small sample sizes). All MG and haplogroup A displayed multimodal and unimodal curves that mismatched distributions, respectively (Figure 4). In particularly, all MG showed two major curves at 11 and 23 pairwise differences with one minor curve at 39 pairwise differences. These results indicate there were at least two population expansion events that occurred in the past. Fu's [32] Fs statistic provided more evidence for past population expansion of MG; this value was significantly negative for all MG and haplogroup A ( $\mathrm{p}<0.001)$ (Figure 4). In addition, SSD and $r$ values were not significant for all MG goats $(p>0.05 ; 0.004$ and 0.003 , respectively), indicating that our total dataset (174 MG) showed relatively good fit to the population expansion model [31].

\section{DISCUSSION}

\section{Genetic diversity}

To date, no study has explored genetic diversity and the origin of MGs in depth. This study conducted the first assessments of genetic population structure, genetic diversity, phylogenetic status, and the origin of Mongolian goats using the most polymorphic HVI region in mtDNA. This highly polymorphic region (indeed, the complete D-loop) has been routinely used

Table 3. Genetic variance of Mongolian native goats (Capra hircus) based on analysis of the hypervariable region 1 region

\begin{tabular}{lcccc}
\hline Source of variation & Degree of freedom & Sum of square & Variance component & Percentage of variation \\
\hline Among population & 11 & 85.73 & 0.095 & 1.46 \\
Within population & 162 & $1,040.98$ & 6.425 & 98.54 \\
Total & 173 & $1,126.71$ & 6.520 & - \\
\hline
\end{tabular}

Fst $=0.014, \mathrm{p}<0.05$. 


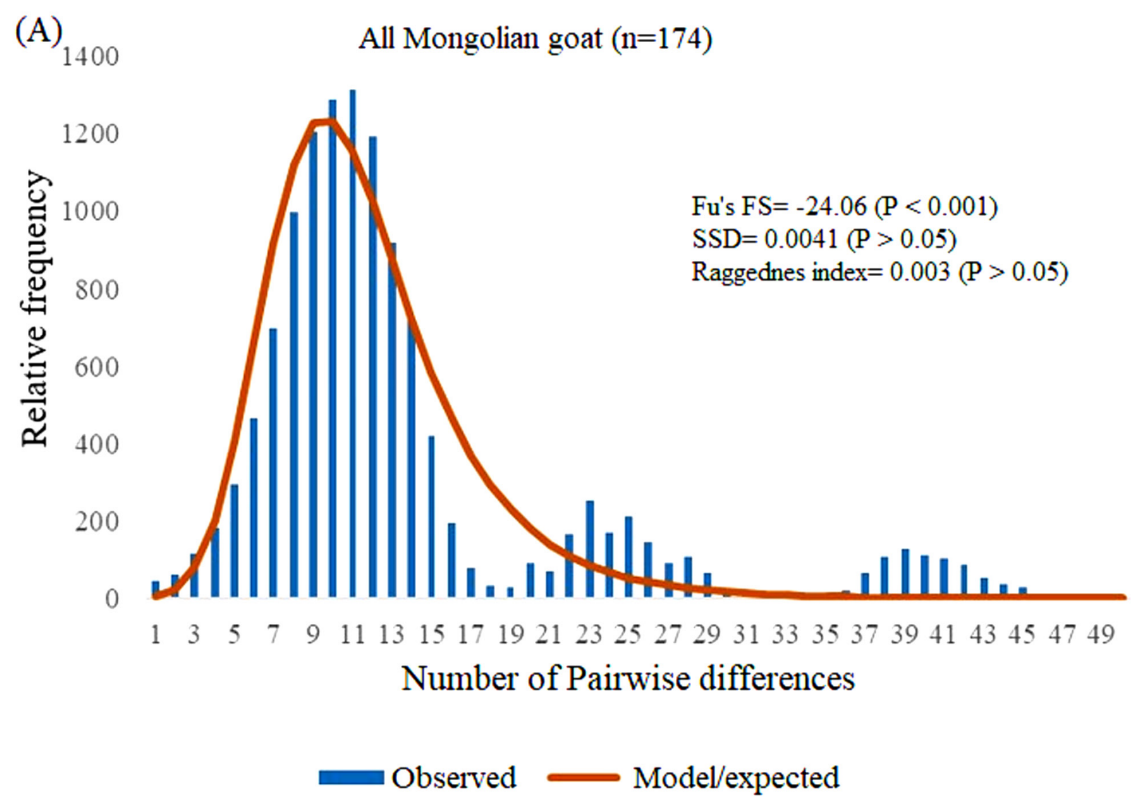

(B)

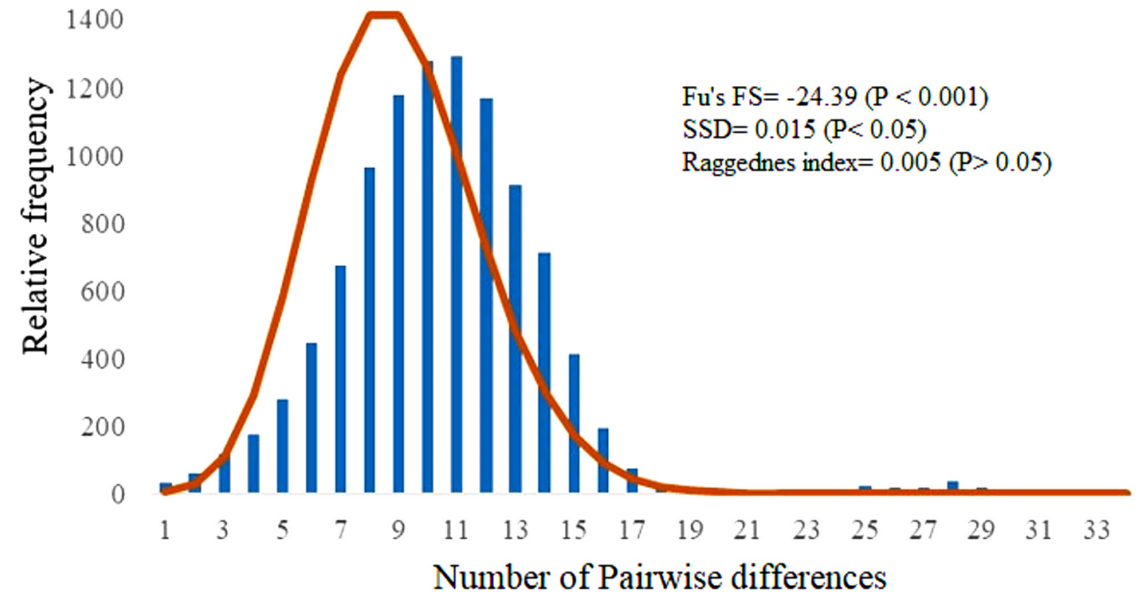

Figure 4. Mismatch distributions and Fu's Fs analysis of $(A)$ all Mongolian goats $(n=174)$ and $(B)$ haplogroup $A(n=158)$.

in previous studies that examined genetic diversity, phylogenetic relationships, and especially the maternal origin of most domesticated animals, including goats [14,33-37].

Similar to previous results $[17,38,39]$, goats from Mongolia investigated in this study showed high mtDNA diversity. The assessed overall $\mathrm{Hd}$ and Pi of MG were 0.997 and 0.0283 , respectively (Table 1 ). Our results also indicated that $H \mathrm{~d}$ in Mongolian goats were slightly higher (not significant) than that found in goat breeds from Africa, Egypt, and south Asia, but significantly higher than in Chinese and European breeds (Supplementary Figure S2A) [17,38,39]. Whereas, Pi values obtained from this study were significantly higher than that found in other regions including domestication center (except Chinese and southern Asian breeds) (Supplementary Figure S2B) $[17,38,39]$. The high level of mtDNA diversity in the world's goat populations was previously explained by multiple maternal effects of wild ancestors [5,6]. Similarly, the high genetic diversity in MG likely resulted from the presence of an abundant haplogroup and crossbred/mixed with foreign populations from the Union of Soviet Socialist Republic (USSR) (Mountain Altai, Don and Unjuul) to improve the quality of their fibers [20].

\section{Maternal origin and phylogeography of Mongolian goats}

As the origin of MG was only initially investigated in this study, these results are far from clear. Mongolians traditionally believed that their goats descended directly from wild Siberian ibex, a species widely distributed in Mongolia [19]. Yet, genetic and archeological studies widely agree that domes- 
tic goats in Mongolia originated from C. aegagrus in Fertile Crescent region of the NE [1,2,6,7]. Meanwhile, Lin et al [22] emphasized that haplogroup B or at least sub-group B1 originated in some region of Southeast Asia. Therefore, all animals that clustered into haplogroup B belong to east or south Asian countries, in particularly sub-group B1 accounted for only Chinese and Mongolian goats in this study (Figures 1B, 3).

The results of our phylogenetic analysis revealed four distinct maternal haplogroups ( $\mathrm{A}, \mathrm{B}, \mathrm{C}$, and $\mathrm{D}$ ) in $\mathrm{MG}$ populations (Figure 1B). Consistent with previous studies $[6,14,15,22,40]$, haplogroup A was predominant (90.8\%) and found in all MG populations. Haplogroup B was found mostly in Asia, but only one individual from the Ref population was found in this haplogroup in this study (Figure 3). Alternatively, haplogroups $\mathrm{C}$ and $\mathrm{D}$ were found from both Asia and Europe, and goats from $\mathrm{G}$ and $\mathrm{F}$ had very limited distributions in Sicily and the NE region, respectively [6]. Some previous studies $[6,14]$ reported that three haplogroups (A, B, and C) occurred in Mongolia based on a very limited sample size. More recently, Lin et al [22] included some Mongolian goats (population's locality unknown) in their diversity analysis. They reported the four haplogroups that we found in this study. To obtain further insight into the origin of MG, NJ tree, MJ network, and estimation of pairwise Fst values were performed based on a combination of goats from Mongolia and those from different regions, including the domestication center (Figures 2, 3). The close relationships of Mongolian and Chinese (from Huanghuai and Leizhou) goats were obtained from MJ network analysis; they shared three haplotypes (H60, H88, H118, Figure 3, Supplementary Table S4) and occurred in $\mathrm{NJ}$ trees' clusters. Interestingly, goat populations $(\mathrm{n}=1)$ from Kazakhstan were very closely related genetically to MG (mean, Fst $=-0.016$ ), and followed by Fst values (mean $=0.103$ ) obtained from comparisons of goat populations ( $\mathrm{n}$ = 5) from some Arabian countries (including the domestication center; i.e. Turkish goat and Baladi breed from Jordan) and $M G$ populations $(n=12)$, this value was lower than that found in comparisons of Chinese $(n=5)$ versus MG populations (0.128), as well as southern Asia ( $\mathrm{n}=4$, mean Fst $=$ 0.275) (Supplementary Table S5). These close relationships among goats from different regions likely resulted from a weak phylogeographic structure of domestic goats, which is likely explained by a high rate of gene flow among populations, even between continents [38].

According to findings from this and previous studies $[6,14$, 22,39], goats from the four haplogroups in Mongolia arrived from two possible domestication centers through ancient human migration or commercial trade routes. We hypothesize that the first domestication center was the NE region for MG from haplogroups $\mathrm{A}, \mathrm{C}$, and $\mathrm{D}$ whose ancient migration routes could be explained by a migration that started in the NE region and passed through central Asia (i.e. Kyrgyzstan and
Kazakhstan, Fst values versus MG was 0.106 and -0.016 , respectively), prior to arriving in Mongolia. This migration may have involved ancient sheep population, because since ancient times Mongolian nomads reared sheep and goat together.

Second, a domestication center was located close to Mongolia somewhere in Southeast Asia [22]. We believe that a smaller group of ancient goats from haplogroup B (B1 subgroup) arrived in Mongolia from this center. It is difficult to determine the date of the first arrival of domestic goats into Mongolia. Nevertheless, archeological findings based on petroglyphs in Mongolia demonstrate that the first goat populations arrived at least 3,000 to 3,500 years ago $[6,41]$. If the first goats arrived with sheep in Mongolia, this dated could be pushed back to 5,000 to 7,000 years ago [10].

\section{Population structure and expansion}

AMOVA analysis of all MG dataset showed very weak phylogeographic structure among 12 populations, and more than $98 \%$ of the total variation occurred within populations. This weak structure was also evidenced by limited genetic differentiations (mean pairwise $F s t=0.0168$ ). Our results were consistent with previous studies $[6,38,42]$. The traditional nomadic culture of Mongolia may have contributed to this weak geographical structure and limited genetic differentiations among MG populations. Under nomadic pasturing, there is a high rate of animal exchanges of the five main types of livestock animals in Mongolia (sheep, horse, cattle, camel, and goat) and often long-distance movements even cross the country.

Mismatch distribution and the neutrality test of Fu's [33] Fs statistics are recognized as suitable approaches to examine past population expansion and were routinely used in previous studies $[6,36,38,39]$. Mismatch distribution is a frequency graph of pairwise differences between alleles, and it has two common patterns, including multimodal (populations at demographic equilibrium) and unimodal (populations passed through a recent demographic expansion) $[29,43]$. Fu's Fs statistic is based on the probability of having a number of alleles equal to or greater than the observed number in a sample drawn from a stationary population [32]. Both approaches revealed at least two population expansions of Mongolian goats in the past: i.e. mismatch distribution pattern was multimodal for all MG while unimodal for haplogroup A, and highly significant and negative Fu's Fs values were observed for all MG (-24.06) and haplogroup A (-24.39). Relatively similar patterns of mismatch distribution and significant and negative Fu's $F s$ values were obtained in previous studies [37, 39,42]. More recently, the number of goats in Mongolia has increased fivefold within the last 30 years, more specifically the goat population grew from 5.1 million in 1990 to 27.3 million by 2017 [18]. 


\section{CONCLUSION}

In summary, we report novel information regarding genetic diversity, population structure, and origin of Mongolian goats. The findings obtained from this study reveal that abundant haplogroups (A to D) occur in goat populations in Mongolia, with high levels of haplotype and Pi. Among these haplogroups, haplogroup A was predominant, with relative frequency of $90.8 \%$. In addition, we speculate that two independent domestication centers may have contributed to the formation of goat populations in Mongolia. Finally, we highlighted that the past nomadic pasturing system led to weak geographical structure and limited genetic differentiations among populations in different regions of Mongolia.

\section{CONFLICT OF INTEREST}

We certify that there is no conflict of interest with any financial organization regarding the material discussed in the manuscript.

\section{ACKNOWLEDGMENTS}

First of all, authors especially thank the herders who agreed to let us collect samples for this study. This study was partially supported by research fund of Chungnam National University in Korea and Mongolian National University of Education.

\section{REFERENCES}

1. Porter V. Goats of the world. Ipswich, UK: Farming Press Limited; 1996.

2. Zeder M, Hesse B. The initial domestication of goats (Capra hircus) in the Zagros Mountains 10,000 years ago. Sciences 2000;287:2254-7.

3. Meadow RH. Animal domestication in the Middle East: a revised view from the eastern margin. In: Possehl G, Oxford and IBH editors. Harappar civilization. New Dehli, India: Harappar Civilization; 1993. p. 295-320.

4. Takada T, Kikkawa Y, Yonekawa H, Kawakami S, Amano T. Bezoar (Capra aegagrus) is a matriarchal candidate for ancestor of domestic goat (Capra hircus): evidence from the mitochondrial DNA diversity. Biochem Genet 1997;35:315-26. https:// doi.org/10.1023/A:1021869704889

5. Mannen H, Nagata Y, Tsuji S. Mitochondrial DNA reveal that domestic goat (Capra hircus) are genetically affected by two subspecies of bezoar (Capra aegagurus). Biochem Genet 2001;39:145-54. https://doi.org/10.1023/A:1010266207735

6. Naderi S, Rezaei HR, Taberlet P, et al. Large-scale mitochondrial DNA analysis of the domestic goat reveals six haplogroups with high diversity. PLoS One 2007;2:p.e1012. https:// doi.org/10.1371/journal.pone.0001012

7. Colli L, Lancioni H, Cardinali I, et al. Whole mitochondrial genomes unveil the impact of domestication on goat matrilineal variability. BMC Genomics 2015;16:1115. https://doi. org/10.1186/s12864-015-2342-2

8. Beja-Pereira A, Caramelli D, Lalueza-Fox C, et al. The origin of European cattle: evidence from modern and ancient DNA. Proc Natl Acad Sci 2006;103:8113-8. https://doi.org/10.1073/ pnas.0509210103

9. Mwacharo JM, Bjørnstad G, Mobegi V, et al. Mitochondrial DNA reveals multiple introductions of domestic chicken in East Africa. Mol Phylogenet Evol 2011;58:374-82. https://doi. org/10.1016/j.ympev.2010.11.027

10.Lv FH, Peng WF, Yang J, et al. Mitogenomic meta-analysis identifies two phases of migration in the history of eastern Eurasian sheep. Mol Biol Evol 2015;32:2515-33. https://doi. org $/ 10.1093 / \mathrm{molbev} / \mathrm{msv} 139$

11. Touma S, Shimabukuro H, Arakawa A, Oikawa T. Maternal lineage of Okinawa indigenous Agu pig inferred from mitochondrial DNA control region. Asian-Australas J Anim Sci 2019;32:501-7. https://doi.org/10.5713/ajas.18.0378

12. Tarekegn GM, Ji XY, Bai X, et al. Variations in mitochondrial cytochrome b region among Ethiopian indigenous cattle populations assert Bos taurus maternal origin and historical dynamics. Asian-Australas J Anim Sci 2018;31:1393-400. https:// doi.org/10.5713/ajas.17.0596

13. Teinlek P, Siripattarapravat K, Tirawattanawanich C. Genetic diversity analysis of Thai indigenous chickens based on complete sequences of mitochondrial DNA D-loop region. AsianAustralas J Anim Sci 2018;31:804-11. https://doi.org/10.5713/ ajas.17.0611

14.Luikart G, Gielly L, Excoffier L, Vigne JD, Bouvet J, Taberlet P. Multiple maternal origins and weak phylogeographic structure in domestic goats. Proc Natl Acad Sci 2001;98:5927-32. https://doi.org/10.1073/pnas.091591198

15. Sultana S, Mannen H. Polymorphism and evolutionary profile of mitochondrial DNA control region inferred from the sequences of Pakistani goats. Anim Sci J 2004;75:303-9. https:// doi.org/10.1111/j.1740-0929.2004.00190.x

16. Sardina MT, Ballester M, Marmi J, et al. Phylogenetic analysis of Sicilian goats reveals a new mtDNA lineage. Anim Genet 2006;37:376-8. https://doi.org/10.1111/j.1365-2052.2006. 01451.x

17.Al-Araimi NA, Al-Atiyat RM, Gaafar OM, et al. Maternal genetic diversity and phylogeography of native Arabian goats. Livest Sci 2017;206:88-94. https://doi.org/10.1016/j.livsci. 2017.09.017

18.NSO (National Statistics Office of Mongolia) [Internet]. Ulaanbaatar, Mongolia: Government III building; 2017 [cited 2018 Sept 2]. Available from: http://www.1212.mn/tables. aspx?TBL_ID=DT_NSO_1001_021V1

19. Ganbold O, Lee SH, Seo D, et al. A review of population gene- 
tics research on domestic animals in Mongolia and recommendations for the improvements. J Anim Breed Genom 2018;2: 9-20. https://doi.org/10.12972/jabng.20180016

20.MLS (Mongolian Livestock Sector) [Internet]. Ulaanbaatar, Mongolia: 2010 [cited 2018 Sept 11]. Available at: https:// mofa.gov.mn/livestock/index.php?option=com_content\&view $=$ category\&layout $=$ blog\&id $=48 \&$ Itemid $=72 \&$ lang $=$ en

21. Ganbold O, Manjula P, Lee SH, et al. Sequence characterization and polymorphism of melanocortin 1 receptor gene in some goat breeds with different coat color of Mongolia. Asian-Australas J Anim Sci 2019;32:939-48. https://doi.org/ 10.5713/ajas.18.0819

22.Lin BZ, Odahara S, Ishida M, et al. Molecular phylogeography and genetic diversity of East Asian goats. Anim Genet 2013; 44:79-85. https://doi.org/10.1111/j.1365-2052.2012.02358.x

23. Hall TA. BioEdit: a user-friendly biological sequence alignment editor and analysis program for Windows 95/98/NT Nucleic Acids Symposium Series, 41. Oxford, UK: Oxford University Press; 1999. pp. 95-8.

24. Thompson JD, Higgins DG, Gibson TJ. CLUSTAL W: improving the sensitivity of progressive multiple sequence alignment through sequence weighting, position-specific gap penalties and weight matrix choice. Nucleic Acids Res 1994;22:467380. https://doi.org/10.1093/nar/22.22.4673

25.Librado P, Rozas J. DnaSP v5: Software for comprehensive analysis of DNA polymorphism data. Bioinformatics 2009; 25:1451-2. https://doi.org/10.1093/bioinformatics/btp187

26. Tamura K, Nei M. Estimation of the number of nucleotide substitutions in the control region of mitochondrial DNA in humans and chimpanzees. Mol Biol Evol 1993;10:512-26. https://doi.org/10.1093/oxfordjournals.molbev.a040023

27. Kumar S, Stecher G, Tamura K. MEGA7: Molecular Evolutionary Genetics Analysis version 7.0 for bigger datasets. Mol Biol Evol 2016;33:1870-4. https://doi.org/10.1093/molbev/ msw054

28. Bandelt HJ, Forster P, Rohl A. Median-joining networks for inferring intraspecific phylogenies. Mol Biol Evol 1999;16: 37-48. https://doi.org/10.1093/oxfordjournals.molbev.a026036

29. Excoffier L, Lischer HEL. Arlequin suite ver 3.5: a new series of programs to perform population genetics analyses under Linux and Windows. Mol Ecol Resour 2010;10:564-7. https:// doi.org/10.1111/j.1755-0998.2010.02847.x

30. Rogers AR, Harpending H. Population growth makes waves in the distribution of pairwise genetic differences. Mol Biol Evol 1992;9:552-69. https://doi.org/10.1093/oxfordjournals. molbev.a040727

31. Harpending HC. Signature of ancient population growth in a low-resolution mitochondrial DNA mismatch distribution. Hum Biol 1994;66:591-600.

32. Fu YX. Statistical tests of neutrality of mutations against population growth, hitchhiking and background selection. Genetics 1997;147:915-25.

33. Hiendleder S. A low rate of replacement substitutions in two major Ovis aries mitochondrial genomes. Anim Genet 1998; 29:116-22. https://doi.org/10.1046/j.1365-2052.1998.00295.x

34. Giuffra EJ, Kijas JMH, Amarger V, Carlborg Ö, Jeon JT, Andersson $\mathrm{L}$. The origin of the domestic pig: independent domestication and subsequent introgression. Genetics 2000;154: 1785-91.

35. Meadows JR, Li K, Kantanen J, et al. Mitochondrial sequence reveals high levels of gene flow between breeds of domestic sheep from Asia and Europe. J Hered 2005;96:494-501. https:// doi.org/10.1093/jhered/esi100

36.Liu J, Ding X, Zeng Y, et al. Genetic diversity and phylogenetic evolution of Tibetan sheep based on mtDNA D-Loop sequences. PloS One 2016;11:e0159308. https://doi.org/10.1002/ ece3.3710

37. Tarekegn GM, Tesfaye K, Mwai OA, et al. Mitochondrial DNA variation reveals maternal origins and demographic dynamics of Ethiopian indigenous goats. Ecol Evol 2018;8:1543-53. https://doi.org/10.1002/ece3.3710

38. Akis I, Oztabak K, Mengi A, Un C. Mitochondrial DNA diversity of A natolian indigenous domestic goats. J Anim Breed Genet 2014;131:487-95. https://doi.org/10.1111/jbg.12096

39.Zhao Y, Zhao R, Zhao Z, Xu H, Zhao E, Zhang J. Genetic diversity and molecular phylogeography of Chinese domestic goats by large-scale mitochondrial DNA analysis. Mol Biol Rep 2014;41:3695-704. https://doi.org/10.1007/s11033-0143234-2

40.Kibegwa FM, Githui KE, Jung'a JO, Badamana MS, Nyamu MN. Mitochondrial DNA variation of indigenous goats in Narok and Isiolo counties of Kenya. J Anim Breed Genet 2016;133:238-47. https://doi.org/10.1111/jbg.12182

41.Ser-odjav N. The archeology of Mongolia. Studies of Archeological Institute and history of Mongolian Academy of Science. 12th ed. Ulaanbaatar, Mongolia: Munkhyn Useg Press; 1987. pp. 31-42.

42.Chen SY, Su YH, Wu SF, Sha T, Zhang YP. Mitochondrial diversity and phylogeographic structure of Chinese domestic goats. Mol Phylogenet Evol 2005;37:804-14. https://doi.org/ 10.1016/j.ympev.2005.06.014

43.Slatkin M, Hudson RR. Pairwise comparisons of mitochondrial DNA sequences in stable and exponentially growing populations. Genetics 1991;129:555-62. 